\title{
Steps towards Quantum Gravity and the practice of science: will the merger of mathematics and physics work?
}

\author{
Bernhelm Booss-Bavnbek
}

\begin{abstract}
I recall general tendencies of the mathematization of the sciences and derive challenges and tentative obstructions for a successful merger of mathematics and physics on fancied steps towards Quantum Gravity. This is an edited version of the opening words to an international workshop Quantum Gravity: An Assessment, Holbæk, Denmark, May 17-18, 2008. It followed immediately after the Quantum Gravity Summer School, see http: / / QuantumGravity.ruc.dk
\end{abstract}

\section{Regarding the need and the chances of unification}

Reading the literature, preparing and attending the Quantum Gravity Summer School, editing of this volume, and discussing with co-editors and contributors provoked the following claims and questions: (1) The natural laboratory for quantum gravity is the universe, of which we have no control. (2) Nevertheless, we have to accept the challenge, and also a new feature: soon a lifetime will be insufficient to verify a new important theory, and only our successors will be able to prove or disprove it. By the way, it took 49 years just for the Casimir effect, let alone Hawking radiation or string theory. (3) Do we really need to unify gravity with the other interactions? Some physicists say "No". However, if gravity stays in isolation, physicists must be afraid that gravitational physics as a subject will die soon. (4) Most innovative work in quantum gravity is balancing on a knife's edge between abstract mathematics and fresh views on physics concepts. A mathematician, however, may have many remaining unanswered questions, both regarding the claims of physics relevance of the most innovative work and regarding the mathematical clearness and reliability of various new concepts and calculations.

B. Booß-Bavnbek

NSM/IMFUFA, Roskilde Universitet, Postboks 260, DK-4000 Roskilde, Denmark, e-mail: boosseruc.dk http://milne.ruc.dk/ Booss/ 
In the following I recall common knowledge on modelling, mathematization, and science history to put the declared "New Paths Towards Quantum Gravity" in a common frame in spite of their scattering and heterogeneity. Some of the following considerations were published in [BEL07] in condensed form before the Summer School as a kind of platform for the assessment of our endeavour. For a comparison with mathematization in other frontier fields I refer also to the recent [Bo09].

\section{The place of physics in John Dee's Groundplat of Sciences and Artes, Mathematicall of 1570}

The use of mathematical arguments, first in pre-scientific investigations, then in other sciences, foremost in medicine and astronomy and in their shared border region astrology, has been traced way back in history by many authors from various perspectives, Bernal [Be54], Høyrup [Ho04], and Kline [K174].

Globally speaking, they all agree on three mathematization tendencies:

1. The progress in the individual sciences makes work on ever more complicated problems possible and necessary.

2. This accumulation of problems and data demands conscious, planned, and economic procedures in the individual sciences, i.e., an increased emphasis on questions of methodology.

3. This increased emphasis on questions of methodology is as a rule associated with the tendency of mathematization.

All of this applies generally. In detail, we find many various pictures. In his Groundplat of Sciences and Artes, Mathematicall of 1570 [De70], the English alchemist, astrologer, and mathematician John Dee, the first man to defend the Copernican theory in Britain and a consultant on navigation, pointed out, in best Aristotelian tradition, that it is necessary in the evaluation of mathematization to pay strict attention to the specific characteristics of the application area in question. He postulated a dichotomy between the Principall side, pure mathematics, and the Deriuative side, i.e., applied mathematics and mathematization. He then classified the applications of pure mathematics according to objects treated:

- Ascending Application in thinges Supernaturall, eternall and Diuine,

- In thinges Mathematicall: Without farther Applications,

and finally, on the lowest and most vulgar plane in the Aristotelian scheme,

- Descending Application in thinges Naturall: both Substantiall \& Accidentall, Visible \& Inuisible \& $c$.

Now that history has excluded matters divine from mathematics, we can with some justification ask whether later generations may regard with equal amusement and 
astonishment the fact that in our time there are a large number of professional mathematicians and physicists, who are completely satisfied with spending their entire lives working in the second, inner mathematical level and who persistently refuse to descend to vulgar applications.

The panorama of the individual sciences and the role that mathematics had to play in them was perfectly clear to John Dee. In our time the matter is somewhat more complex. For a class on Quantum Gravity, I cannot point out a geodetically perfect picture of today's landscape of mathematization nor of precise border lines between the mathematics and physics addressed. I must treat the matter rather summarily. A summary treatment may have the advantage that in comparison among the mathematization progress in different branches of physics, common problems on one hand and on the other hand special features of the here advocated new paths towards quantum gravity can be seen more clearly.

In the following, I shall restrict myself to the study of dead nature in physics, the field which has the highest degree of mathematization on any chosen scale, both quantitatively and qualitatively. To put things in relief, I shall occasionally touch upon the investigation of living matter in medicine, the field where one might expect the greatest mathematization advances in our century, and confront our highly speculative branch of mathematical physics with the treatment of financial issues and decision making for commerce and production in economics, a field of questionable scientific state, that, beyond well founded actuary estimations, lacks unambiguous results and convincing clear perspectives regarding mathematization.

\section{Delimitation between mathematics and physics}

The intimate connection between mathematics and physics makes it difficult to determine the theoretical relevance of mathematics and obscures the boundary between genuinely physical thought and observation on one side and the characteristically mathematical contribution on the other side. Recall Hilbert's perception of probability theory as a chapter of physics in his famous 6th Problem [Hi00]:

6. Mathematical Treatment of the Axioms of Physics. The investigations on the foundations of geometry suggest the problem: To treat in the same manner, by means of axioms, those physical sciences in which already today mathematics plays an important part; in the first rank are the theory of probabilities and mechanics.

To say it mildly, as Gnedenko did in his comments to the Russian edition of 1969: Today this viewpoint (to consider probability theory as a chapter of physics) is no longer so common as it was around the turn of the century, since the independent mathematical content of the theory of probabilities has sufficiently clearly showed since then... With hindsight and in view of the still challenging foundational problems of quantum mechanics, however, we may accept that parts of mathematics and physics can be interlaced in a non-separable way.

Another famous example of that inextricable interlacement is provided by the Peierls-Frisch memorandum of 1940 to the British Government: suggested by the 
codiscoverer of fission Otto Frisch, the physicist Rudolph Peierls, like Frisch a refugee in Britain, made the decisive feasibility calculation that not tons (as - happily - erroneously estimated by Heisenberg in the service of the Nazis) but only about $1 \mathrm{~kg}$ (later corrected to $6 \mathrm{~kg}$ ) of the pure fissile isotope $U_{235}$ would be needed to make the atomic bomb. Was it mathematics or physics? It may be worth mentioning that Peierls was a full professor at the University of Birmingham since 1937 and became joint head of mathematics there, [Ed96]. Theoretical physics in Britain is often in mathematics. As a matter of fact, physics in our sense did not exist as a single science before the nineteenth century. There were well-defined experimental physics comprising heat, magnetism, electricity and colour, leaving mechanics in mathematics, see [Ho04, p. 493].

In spite of that intermingling, physics can provide a ready system of categories to distinguish different use of mathematics in different modelling situations. Perhaps, the situation can be best compared with the role of physics in general education. After all, physics appears as the model of mathematization: there is no physics without mathematics - and, as a matter of fact, learning of mathematics is most easy in a physics context: calculation by letters; the various concepts of a function (table, graph, operation) and its derivatives and anti-derivatives; differential equations; the concept of observational errors and the corresponding estimations and tests of hypotheses; Brownian movements; all these concepts can be explained context-free or in other contexts (where some of the concepts actually originated), but they become clearest in the ideally simple applications of physics, which are sufficiently complicated to see the superiority of the mathematization as compared to feelings, qualitative arguments, discussions, convictions, imagination - but simple enough to get through.

\section{Variety of modelling purposes}

It may be helpful to distinguish the following modelling purposes:

\subsection{Production of data, model-based measurements}

Clearly, the public associates the value of mathematical modelling foremost to its predictive power, e.g., in numerical weather prediction, and its prescriptive power, e.g., in the design of the internal ballistics of the hydrogen bomb; more flattering to mathematicians, the explanatory power of mathematization and its contribution to theory development yield the highest reputation within the field. However, to the progress of physics, the descriptive role, i.e., supporting model-based measurements in the laboratory, is - as hitherto - the most decisive contribution of mathematics. Visco-elastic constants and phase transition processes of glasses and other soft materials can not be measured directly. For high precision in the critical region, one 
measures electric currents through a "dancing" piezoelectric disc with fixed potential and varying frequency. In this case, solving mathematical equations from the fields of electro-dynamics and thermo-elasticity becomes mandatory for the design of the experiments and the interpretation of the data. In popular terms, one may speak of a mathematical microscope, in technical terms of a transducer that becomes useful as soon as we understand the underlying mathematical equations.

\subsection{Simulation}

Once a model is found and verified and the system's parameters are estimated for one domain, one has the hope of doing computer calculations to predict what new experiments in new domains (new materials, new temperatures etc) should be made and what they might be expected to show. Rightly, one has given a special name of honour to that type of calculations, computer simulations: as a rule, it requires to run the process on a computer or a network of computers under quite sophisticated conditions: typically, the problem is to bring the small distances and time intervals of well-understood molecular dynamics up to reasonable macroscopic scales, either by aggregation or by Monte Carlo methods - as demonstrated by Buffon's needle casting for the numerical approximation of $\pi$.

One should be aware that the word simulation has, for good and bad, a connotation derived from NASA's space simulators and Nintendo's war games and jukeboxes. Animations and other advanced computer simulations can display an impressive beauty and convincing power. That beauty, however, is often their dark side: simulations can show a deceptive similarity with true observations, so in computational fluid dynamics when the numerical solution of the Bernoulli equations, i.e., the linearization of the Navier-Stokes equations for laminar flow displays eddies characteristic for the non-linear flow. The eddies do not originate from real energy loss due to friction and viscosity but from hardly controllable hardware and software properties, the chopping of digits, thus providing a magic realism, as coined by Abbott and Larsen [AbLa85]. In numerical simulation, like in mathematical statistics, results which fit our expectations too nicely, must awake our vigilance instead of being taken as confirmation.

\subsection{Prediction}

As shown in the preceding subsection, there is no sharp boundary between description and prediction. However, the quality criteria for predictions are quite simple: do things develop and show up as predicted? So, for high precision astrology and longitudinal determination in deep-sea shipping the astronomical tables of planetary movement, based on the outdated and falsified Ptolemaic system (the Resolved Alfonsine Tables) and only modestly corrected in the Prutenic Tables of 1551 were, 
until the middle of the 17th century, rightly considered as more reliable than Kepler's heliocentric Rudolphine Tables, as long as they were more precise - no matter on what basis, see, Steele [St00 p. 128].

Almost unnoticed, we have had a similar revolution in weather prediction in recent years: the (i) analogy (synoptic) methods of identifying a similarly looking weather situation in the weather card archives to base the extrapolation on it were replaced by almost pure (ii) numerical methods to derive the prediction solely from the thermodynamic and hydrodynamic basic equations and conservation laws, applied to initial conditions extracted from the observation grid. "Almost" because the uncertainty of the interpolation of the grid and the high sensitivity of the evolution equations to initial conditions obliges to repeated runs with small perturbations and human inspection and selection of the most "probable" outcome like in (i). That yields sharp estimates about the certainty of the prediction for a range of up to 10 days. In nine of ten cases, the predictions are surprisingly reliable and would have been impossible to obtain by traditional methods. However, a $10 \%$ failure rate would be considered unacceptable in industrial quality control.

In elementary particle physics, the coincidence of predictions with measurements is impressive, but also disturbing. I quote from Smolin [Sm06, pp. 12-13]:

Twelve particles and four forces are all we need to explain everything in the known world. We also understand very well the basic physics of these particles and forces. This understanding is expressed in terms of a theory that accounts for all these particles and all of the forces except for gravity. It's called the standard model of elementary-particle physics - or the standard model for short. ... Anything we want to compute in this theory we can, and it results in a finite number. In the more than thirty years since it was formulated, many predictions made by this theory have been checked experimentally. In each and every case, the theory has been confirmed.

The standard model was formulated in the early 1970s. Except for the discovery that neutrinos have mass, it has not required adjustment since. So why wasn't physics over by 1975 ? What remained to be done?

For all its usefulness, the standard model has a big problem: It has a long list of adjustable constants. ...

We feel pushed back to the pre-Keplerian, pre-Galilean and pre-Newtonian cosmology built on ad-hoc assumptions, displaying clever and deceptive mathematicsbased similarity between observations and calculations - and ready to fall at any time because the basic assumptions are not explained.

Perhaps the word deceptive is inappropriate when speaking of description, simulation and prediction: for these tasks, similarity can rightly be considered as the highest value obtainable, as long as one stays in a basically familiar context. From a semiotic angle, the very similarity must have a meaning and is indicating something; from a practical angle, questions regarding the epistemological status can often be discarded as metaphysical exaggerations: who cares about the theoretical or ad-hoc basis of a time schedule in public transportation - as long as the train leaves on time! 


\subsection{Control}

The prescriptive power of mathematization deserves a more critical examination. In physics and engineering we may distinguish between the (a) feasibility, the (b) efficiency, and the (c) safety of a design. A design can be an object like an airplane or a circuit diagram for a chip, an instrument like a digital thermometer, TV set, GPS receiver or pacemaker, or a regulated process like a feed-back regulation of the heat in a building, the control of a power station or the precise steering of a radiation canon in breast cancer therapy. Mathematics has its firm footing for testing (a) in thought experiments, estimations of process parameters, simulations and solving equations. For testing (b), a huge inventory is available of mathematical quality control and optimization procedures by variation of key parameters.

It seems to me, however, that (c), i.e., safety questions provide the greatest mathematical challenges. They appear differently in (i) experience-based, (ii) sciencebased and (iii) science-integrated design. In (i), mathematics enters mostly in the certification of the correctness of the design copy and the quality test of the performance. In (ii), well-established models and procedures have to be modified and re-calculated for a specific application. Experienced physicists and engineers, however, seldom trust their calculations and adaptations. Too many parameters may be unknown and pop-up later: Therefore, in traditional railroad construction, a small bridge was easily calculated and built, but then photogrammetrically checked when removing the support constructions. A clash of more than $\delta_{\text {crit }}$ required re-building. Similarly, even the most carefully calculated chemical reactors and other containers under pressure and heat have their prescribed "Soll-Bruchstelle" (supposed line of fracture) in case that something is going wrong.

The transition from (ii) to (iii) is the most challenging: very seldom one introduces a radically new design in the physics laboratory or engineering endeavour. But there are systems where all components and functions can be tested separately though the system as a whole can only be tested in situ: a new design of a Diesel ship engine; a car, air plane or space craft; a new concept in cryptography. In all these cases, one is tempted to look and even to advocate for mathematical proofs of the safe function according to specification. Unfortunately, in most cases these "proofs" belong rather to the field of fiction than to rigorous mathematics. For an interesting discussion on "proofs" in cryptography (a little remote from physics) see the debate between Koblitz and opponents in [Ko08] and follow-ups in the Notices of the American Mathematical Society.

An additional disturbing aspect of science-integrated technology development is the danger of a loss of transparency. Personally, I must admit, I am grateful for most black-box systems. I have no reason to complain when something in my computer is hidden for my eyes, as long as everything functions as it shall or can easily be re-tuned. However, for the neighbourhood of a chemical plant (and the reputation of the company) it may be better not to automatize everything but to keep some aspects of the control non-mathematized and in the hands of the service crew to avoid dequalification and to keep the crew able to handle non-predictable situations. 
A last important aspect of the prescriptive power of the mathematization is its formatting power for thought structure and social behaviour. It seems that there is not so much to do about it besides being aware of the effects.

\subsection{Explain phenomena}

The noblest role of mathematical concepts in physics is to explain phenomena. Einstein did it when reducing the heat conduction to molecular diffusion, starting from the formal analogy of Fick's Law with the cross section of Brownian motion. He did it also when generalizing the Newtonian mechanics into the special relativity of constant light velocity and again when unifying forces and curvature in general relativity.

Roughly speaking, mathematical models can serve physics by reducing new phenomena to established principles; as heuristic devices for suitable generalizations and extensions; and as "a conceptual scheme in which the insights ... fit together" (C. Rovelli). Further below I shall return to the last aspect - the unification hope.

Physics history has not always attributed the best credentials to explaining phenomena by abstract constructions. It has discarded the concept of a ghost for perfect explanation of midnight noise in old castles; the concept of ether for explaining the finite light velocity; the phlogiston for burning and reduction processes, the Ptolemaic epicycles for planetary motion. It will be interesting to see in the years to come whether the mathematically advanced String Theory or the recent Connes-Marcolli reformulation of the Standard Model in terms of spectral triples will undergo the same fate.

\subsection{Theory development}

Finally, what has been the role of mathematical concepts and mathematical beauty for the very theory development in physics? One example is Johann Bernoulli's purely aesthetic confirmation of Galilean fall law $s=g / 2 t^{2}$ among a couple of candidates as being the only one providing the same equation (shape) for his brachistochrone and Huygens' tautochrone, [Be97, p. 395]:

Before I end I must voice once more the admiration that I feel for the unexpected identity of Huygens tautochrone and my brachistochrone. I consider it especially remarkable that this coincidence can take place only under the hypothesis of Galilei, so that we even obtain from this a proof of its correctness. Nature always tends to act in the simplest way, and so it here lets one curve serve two different functions, while under any other hypothesis we should need two curves.

Another, more prominent example is the lasting triumph of Maxwell's equations: a world of radically new applications was streaming out of the beauty and simplicity of the equations of electro-magnetic waves! 
However, not every mathematical, theoretical and empirical accumulation leads to theory development. Immediately after discovering the high-speed rotation of the Earth around its own axis, a spindle shape of the Earth was suggested and an infinitesimal tapering towards the North pole confirmed in geodetic measurements around Paris. Afterwards, careful control measurements of the gravitation at the North Cap and at the Equator suggested the opposite, namely an ellipsoid shape with flattened poles. Ingenious mathematical mechanics provided a rigorous reason for that. Gauss and his collaborator Listing, however, found something different in their control. They called the shape gleichsam wellenförmig and dropped the idea of a theoretically satisfactory description. Since then we speak of a Geoid. For details see Listing [Li73] and the receent Torge [To01, p. 3].

\section{5 "The trouble with physics"}

That is the title of an interesting and well-informed polemic by Lee Smolin against String Theory and present main stream physics at large. He notices a stagnation in physics, so much promise, so little fulfillment [Sm06. p. 313], a predominance of anti-foundational spirit and contempt for visions, partly related to the mathematization paradigm of the 1970s, according to Smolin: Shut up and calculate.

Basically, Smolin may be right. Børge Jessen, the Copenhagen mathematician and close collaborator of Harald Bohr once suggested to distinguish in sciences and mathematics between periods of expansion and periods of consolidation. Clearly physics had a consolidation period in the first half of the 20th century with relativity and quantum mechanics. The same may be true for biology with the momentous triumph of the DNA disclosure around 1950, while, to me, the mathematics of that period is characterized by an almost chaotic expansion in thousands of directions. Following that way of looking, mathematics of the second half of the 20th century is characterized by an enormous consolidation, combining so disparate fields like partial differential equations and topology in index theory, integral geometry and probability in point processes, number theory, statistical mechanics and cryptography, etc. etc. A true period of consolidation for mathematics, while - at least from the outside - one can have the impression that physics and biology of the second half of the 20th century were characterized merely by expansion, new measurements, new effects - and almost total absence of consolidation or, at least failures and vanity of all trials in that direction.

Indeed, there have been impressive successes in recent physics, in spite of the absence of substantial theoretical progress in physics: perhaps the most spectacular and for applications most important discovery has been the High Temperature Superconducting (HTS) property of various ceramic materials by Bednorz and Müller seemingly without mathematical or theoretical efforts but only by systematic combinatorial variation of experiments - in the tradition of the old alchemists, [BeMu87]. 
The remarkable advances in fluid dynamics, weather prediction, oceanography, climatic modelling are mainly related to new observations and advances in computer power while the equations have been studied long before.

Nevertheless, I noticed a turn to theory among young experimental physicists in recent years, partly related to investigating the energy landscapes in material sciences, partly to the re-discovery of the interpretational difficulties of quantum mechanics in recent quantum optics.

\section{Theory - model - experiment}

Physics offers an extremely useful practical distinction between theory, model and experiments. From his deep insight in astronomy, computing, linguistics and psychology, Peter Naur ridicules such distinctions as "metaphysical exaggeration" in [Na95]. He may be right. We certainly should not exaggerate the distinction. In this review, however, the distinction helps to focus on differences of the role of mathematics in doing science.

\subsection{First principles}

By definition, the very core of modelling is mathematics. Moreover, if alone by the stochastic character of observations, but also due to the need to understand the mathematics of all transducers involved in measurements, mathematics has its firm stand with experiments. First principles, however, have a different status: they do not earn their authority from the elegance of being mathematically wrapped, but from the almost infinite repetition of similar and, as well disparate observations connected to the same principle(s). In the first principles, mathematics and physics meet almost on eye level: first principles are also established - like mathematics, and are only marginally questioned. To me, the problem with the pretended eternal authority of first principles is that new cosmological work indicates that the laws of nature may also have undergone some development; that there might have "survived" some evolutionary relics; and that we had better be prepared to be confronted under extreme experimental conditions, with phenomena and relations which fall out of the range of accredited first principles. The canonical candidate for such a relic is the Higgs particle, whether already observed or not. Participants of the Quantum Gravity Assessment Workshop 2008 will recall Holger Bech Nielsen's contributions. 


\subsection{Towards a taxonomy of models}

Not necessarily for the credibility of mathematical models, but for the way of checking the range of credibility, the following taxonomy of models may be extremely useful.

The Closing Round Table of the International Congress of Mathematicians (Madrid, August 22-29, 2006) was devoted to the topic Are pure and applied mathematics drifting apart? As a panelist, Yuri Manin subdivided the mathematization, i.e., the way mathematics can tell us something about the external world, into three modes of functioning (similarly Bohle, Booß and Jensen 1983, [BBJ84], see also [Bo91]):

1. An (ad-hoc, empirically based) mathematical model "describes a certain range of phenomena, qualitatively or quantitatively, but feels uneasy pretending to be something more." Manin gives two examples of the predictive power of such models, Ptolemy's model of epicycles describing planetary motions of about 150 BCE, and the standard model of around 1960 describing the interaction of elementary particles, besides legions of ad-hoc models which hide the lack of understanding behind a more or less elaborated mathematical formalism of organizing available data.

2. A mathematically formulated theory is distinguished from an ad-hoc model primarily by its "higher aspirations. A theory, so to speak, is an aristocratic model." Theoretically substantiated models, such as Newton's mechanics, are not necessarily more precise than ad-hoc models; the coding of experience in the form of a theory, however, allows a more flexible use of the model, since its embedding in a theory universe permits a theoretical check of at least some of its assumptions. A theoretical assessment of the precision and of possible deviations of the model can be based on the underlying theory.

3. A mathematical metaphor postulates that "some complex range of phenomena might be compared to a mathematical construction". As an example, Manin mentions artificial intelligence with its "very complex systems which are processing information because we have constructed them, and we are trying to compare them with the human brain, which we do not understand very well - we do not understand almost at all. So at the moment it is a very interesting mathematical metaphor, and what it allows us to do mostly is to sort of cut out our wrong assumptions. If we start comparing them with some very well-known reality, it turns out that they would not work."

Clearly, Manin noted the deceptive formal similarity of the three ways of mathematization which are radically different with respect to their empirical foundation and scientific status. He expressed concern about the lack of distinction and how that may "influence our value systems". In the words of [Bo91, p. 73]:

Well founded applied mathematics generates prestige which is inappropriately generalized to support these quite different applications. The clarity and precision of the mathematical derivations here are in sharp contrast to the uncertainty of the underlying relations assumed. In fact, similarity of the mathematical formalism involved tends to mask the differences in the scientific extra-mathematical status, in the credibility of the conclusions and in appropriate ways of checking assumptions 
and results... Mathematization can - and therein lays its success - make existing rationality transparent; mathematization cannot introduce rationality to a system where it is absent... or compensate for a deficit of knowledge.

Asked whether the last 30 years of mathematics' consolidation raise the chance of consolidation also in phenomenologically and metaphorically expanding sciences, Manin hesitated to use such simplistic terms. He recalled the notion of Kolmogorov complexity of a piece of information, which is, roughly speaking,

the length of the shortest programme, which can be then used to generate this piece of information... Classical laws of physics - such phantastic laws as Newton's law of gravity and Einstein's equations - are extremely short programmes to generate a lot of descriptions of real physical world situations. I am not at all sure that Kolmogorov's complexity of data that were uncovered by, say, genetics in the human genome project, or even modern cosmology data ... is sufficiently small that they can be really grasped by the human mind.

\subsection{The scientific status of quantum gravity as compared to medicine and economics}

From the rich ancient literature preserved, see Diepgen [Di49], Kudlien [Ku67] and, in particular, Jürss [Ju82, 312-315], we can see that the mind set in Greek medicine already from the fifth century BCE was ours: instead of the partition (familiar from earlier and shaman medicine and similar to the mind set preserved, as seen above, in physics until recent times) into an empirical-rational branch (healing wounds) and a religious-magic branch (cure inner diseases), a physiological concept emerged which focused on the patient as an individual organism within a population, with organs, liquids and tissue, subjected to environmental and dietetic influences and, in principle, open for unconfined investigation of functions, causal relations and the progressive course of diseases. In Hippocratic medicine, we meet for the first time the visible endeavour after a rational surmounting of all problems related to body events.

With a shake of the head, we may read of Greek emphasis and speculations about the body's four liquids or other strange things, like when we recall today the verdict of the medical profession 60 years ago against drinking water after doing sports and under diarrhoea or their blind trust in antibiotics, not considering resistance aspects at all. Admittedly, we have no continuity of results in medicine, but, contrary to physics, we have an outspoken continuity in mind set: no ghosts, no metaphysical spirits, no fancied particles or relations are permitted to enter our explanations, diagnoses, prevention, cure and palliation.

Physicists of our time like to date the physics' beginning back to Galileo Galilei and his translation of measurable times and distances on a skew plane into an abstract fall law. Before Galilei - and long time after him, the methodological scientific status of what we would call mechanical physics was quite low as compared 
with medicine. Physics was a purely empirical subject. It was about precise series of observations and quantitative extrapolations. It was the way to predict planetary positions, in particular eclipse times, the content of silver in compounds, or the manpower required to lift a given weight with given weight arm. It was accompanied and mixed up with all kinds of speculations about the spirits and ghosts at work. We can easily see the continuity of results, of observations and calculations from Kepler and Newton to our time. However, we can hardly recognize anything in their thinking about physics, in the way they connected physics with cosmic music or alchemy or formulated assumptions. We may wonder what later generations will think about our fancied new paths towards quantum gravity.

While a rational point of departure for economics, in particular under the present crisis, can only be a systems view, a holistic unifying view in physics like our efforts in quantum gravity have a smell of vanity. One may argue that the time has hardly come for that endeavour - comparable to the felt necessity but still continuing futility of or at least doubts about a holistic all-embracing systems biology programme in medicine.

\section{General trends of mathematization and modelling}

\subsection{Deep divide}

Regarding the power and the value of mathematization, there is a deep moral divide both within the mathematics community and the public.

On the one side, we have the outspoken science and math optimism of outstanding thinkers: Henri Poincaré's Nature not only suggests to us problems, she suggests their solution; David Hilbert's Wir müssen wissen; wir werden wissen - We must know; we will know of his Speech in Königsberg in 1930, now on his tomb in Göttingen; or Bertolt Brecht's vision of mathematical accountability in Die Tage der Kommune [Br45] of 1945: "Das ist die Kommune, das ist die Wissenschaft, das neue Jahrtausend... - That is the Commune, that is the science, the new millennium..."). We have astonishing evidence that many mathematization concepts either appear to us as natural and a-priori, or they use to emerge as clear over time. We have the power and validity of extremely simple concepts, as in dimension analysis, consistency requirements and gauge invariance of mathematical physics. Progressive movements emphasize science and education in liberation movements and developing countries. Humanitarian organizations (like WHO and UNICEF) preach science and technology optimism in confronting mass poverty and epidemics.

On the other side, deep limitation layers of science and mathematical thinking have been dogged up by Kurt Gödel's Incompleteness Theorem for sufficiently rich arithmetic systems, Andrei N. Kolmogorov's Complexity Theory, and Niels Bohr's notion of Complementarity. Incomprehensibility and lack of regularity continue to hamper trustworthy mathematization. Peter Lax [La08, p. 142] writes about the pro- 
found mystery of fluids, though recognizing that different approaches lead to remarkable coincidence results, supporting reliability.

The abstruseness of the mathematical triumphs of the hydrogen bomb is commonplace. The wide-spread trust in superiority and invincibility, based on mathematical war technology like high precision bombing, has proved to be even more vicious for warriors and victims than the immediate physical impact of the very math-based weaponry, recently also in Iraq and Afghanistan.

In between the two extremes, Hilbert's optimistic prediction of clearness and the sceptical Kafkaesque expectation of increasing bewilderment when digging deeper mathematically, we have the optimistic scepticism of Eugene Wigner's unreasonable effectiveness of mathematics, but also Jacob Schwartz's verdict against the pernicious influence of mathematics on science and Albert Einstein's demand for finding the central questions against the dominance of the beautiful and the difficult.

\subsection{Charles Sanders Peirce's semiotic view}

From the times of Niels Bohr, many physicists, mathematicians and biologists have been attentive to philosophical aspects of our doing. Most of us are convinced that the frontier situation of our research can point to aspects of some philosophical relevance - if only the professional philosophers would take the necessary time to become familiar with our thinking. Seldom, however, we read something of the philosophers which can inspire us.

The US-American philosopher Charles Sanders Peirce (1839-1914) is an admirable exception. In his semiotics and pragmaticist (he avoids the word "pragmatic") thinking, he provides a wealth of ideas, spread over an immense life work. It seems to me that many of his ideas, comments, and concepts can shed light on the why and how of mathematization. Here I shall only refer some thoughts of Peirce's The Fixation of Belief from 1877, see [Pe77].

My fascination of Peirce's text is, in particular, based on the following observations which may appear trivial (or known from Friedrich Engels), but are necessary to repeat many times for the new-modeller:

1. For good and bad, we are all equipped with innate (or spontaneous) orientation, sometimes to exploit, sometimes to subdue. Our innate orientation is similar to the habits of animals in our familiar neighbourhood. We are all "logical machines".

2. However, inborn logic is not sufficient in foreign (new) situations. For such situations, we need methods how to fixate our beliefs. Peirce distinguishes four different methods. All four have mathematical aspects and are common in mathematical modelling.

Tenacity is our strength not to become confused, not to be blown away by unfounded arguments, superficial objections, misleading examples, though sometimes keeping our ears locked for too long. 
Authority of well-established theories and results is what we tend to believe in and have to stick to. We will seldom drop a mastered approach in favour of something new and unproved.

Discussion can hardly help to overcome a belief built on tenacity or authority.

Consequences have to be investigated in all modelling. At the end of the day, they decide whether we become convinced of the validity of our approach (Peirce's Pragmaticist Maxim).

3. The main tool of modelling (i.e., the fixation of belief by mathematical arguments) is the transformation of symbols (signals, observations, segments of reality) into a new set of symbols (mathematical equations, models and descriptions). The advantage for the modeller, for the person to interpret the signs, is that signs which are hard or humid and difficult to collect in one hand can be replaced by signs which we can write and manipulate.

4. The common mapping cycle reality $\rightarrow$ model $\rightarrow$ validation is misleading. The quality of a mathematical model is not how similar it is to the segment of reality under consideration, but whether it provides a flexible and goal-oriented approach, opening for doubts and indicating ways for the removal of doubts (later trivialized by Popper's falsification claim). More precisely, Peirce claims

- Be aware of differences between different approaches!

- Try to distinguish different goals (different priorities) of modelling as precise as possible!

- Investigate whether different goals are mutually compatible, i.e., can be reached simultaneously!

- Behave realistically! Don't ask: How well does the model reflect a given segment of the world? But ask: Does this model of a given segment of the world support the wanted and possibly wider activities / goals better than other models?

I may add: we have to strike a balance between Abstraction vs. construction, Top-down vs. bottom-up, and Unification vs. specificity. We better keep aware of the variety of Modelling purposes and the multifaceted relations between Theory model - experiment. Our admiration for the Power of mathematization, the Unreasonable effectiveness of mathematics (Wigner) should not blind us for the Staying and deepening limitations of mathematization opposite new tasks.

\section{References}

AbLa85. Michael Abbott, Jesper Larsen, Jianhua Tao, Modelling circulations in depth-averaged flows, Part 1: The accumulation of the evidence, J. Hydr. Res. 23 (1985), 309-326; Michael Abbott, Jesper Larsen, Modelling circulations in depth-averaged flows, Part 2: A reconciliation, J. Hydr. Res. 23 (1985), 397-420.

Ba07. John Baez, This Week's Finds, Work on quantum gravity has seemed stagnant and stuck for the last couple of years, which is why I've been turning more towards pure math, Week 220 (August 31, 2005), http://math.ucr.edu/home/baez/week220.html 
BeMu87. J. Georg Bednorz and K. Alex Müller, Perovskite-type oxides The new approach to high- $T_{c}$ superconductivity, Nobel Lecture, December 8, 1987; also in Gösta Ekspong (ed.), Nobel Lectures in Physics 1981-1990, World Scientific Publishing Company, Singapore, 1993.

Be54. John Desmond Bernal, Science in History, 4 vols, 1328 p., Watts, London, 1954.

Be97. Johann Bernoulli, Acta Eruditorum May 1697, 201-224, translated in excerpts in Dirk Struik (ed.), Source Book in Mathematics, 1200-1800, Princeton University Press, Princeton, 1986.

BBJ84. Martin Bohle-Carbonell, Bernhelm Booß, Jens Højgaard Jensen, Innermathematical vs. extramathematical obstructions to model credibility, in: Avula, X. (ed.), Mathematical Modelling in Science and Technology, Proceedings of the Fourth Internat. Conf. (Zürich, August 1983), Pergamon Press, New York, 1984, pp. 62-65.

Bo79. Bernhelm Booß, Niakoi tendenzii v matematishaziiata na otdelnite nauki (Some tendencies in the mathematization of the individual sciences, Bulgarian), Fiz.-Mat. Spis. B"lgar. Akad. Nauk. 22(55), no. 3 (1979), 197-204.

Bo91. Bernhelm Booß-Bavnbek, Against ill-founded, irresponsible modelling, in: Niss, M. et al. (eds.), Teaching of Mathematical Modelling and Applications, Ellis Horwood, Chichester, 1991, pp. 70-82.

Bo09. Bernhelm Booß-Bavnbek, The mathematization of the individual sciences - revisited, Journal of Contemporary Mathematical Analysis (Armenian Academy of Sciences 44/2 (April 2009), 81-96, arXiv:0903.0942v1 [math.HO]. Russian edition: Izvestiya NAN Armenii. Matematika 44/2 (April 2009), 17-38.

BEL07. Bernhelm Booß-Bavnbek, Giampiero Esposito, Matthias Lesch, Quantum gravity: unification of principles and interactions, and promises of spectral geometry, SIGMA (Symmetry, Integrability and Geometry: Methods and Applications) 3 (2007), 098-127, arXiv:0708.1705v3 [hep-th].

Br45. Bertolt Brecht, Die Tage der Kommune, in: Stücke 8, Aufbau-Verlag, Berlin and Weimar, Suhrkamp Verlag, Frankfurt, 1992, pp. 243-317, here p. 174.

De70. John Dee, Mathematicall Praeface to the Elements of Geometrie of Euclid of Megara, 1570. Kessinger Publishing, 1999, 60 pages.

Di49. Paul Diepgen, Geschichte der Medizin. Die historische Entwicklung der Heilkunde und des ärztlichen Lebens, vol. 1, Walter de Gruyter \& Co., Berlin, 1949, pp. 67-158.

Ed96. Sam Edwards, Rudolph E. Peierls - Obituary, Physics Today 49/2 (February 1996), 74-75.

HaK108. Babak Haghighat, Albrecht Klemm, Topological Strings on Grassmannian Calabi-Yau manifolds, JHEP 0901:029-65, 2009, arXiv:0802.2908 1 [hep-th]

Hi00. David Hilbert, Mathematische Probleme, Vortrag, gehalten auf dem internationalen Mathematiker-Kongreß zu Paris 1900, Nachrichten von der Königl. Gesellschaft der Wissenschaften zu Göttingen. Mathematisch-Physikalische Klasse (1900), 253-297; http://www.mathematik.uni-bielefeld.de/ kersten/hilbert/rede.html with Boris Gnedenko's comments to the 6th problem in: P. S. Alexandrov (ed.), Problemi Gilberta, Nauka, Moscow, 1969, German translation in Ostwalds Klassiker der exakten Wissenschaften, vol. 252, Akademische Verlagsgesellschaft, Leipzig, 1971, pp. $145-150$.

Ho04. Jens Høyrup, From Hesiod to Saussure, from Hippocrates to Jevons An Introduction to the History of Scientific Thought, Roskilde 2004; http://akira.ruc.dk/\$ \\sim\}\$jensh/Publications/FromHesiod_04.pdf

Ju82. Fritz Jürss, Geschichte des wissenschaftlichen Denkens im Altertum, Akademie-Verlag, Berlin, 1982.

K174. Morris Kline, Mathematical Thought from Ancient to Modern Times, Oxford University Press, 1974

Ko08. Noel Koblitz, The uneasy relationship between mathematics and cryptography, Notices AMS 54/8 (September 2007), 972-979;

http://www.ams.org/notices/200708/tx070800972p.pdf


Ku67. Fridolf Kudlien, Der Beginn des medizinischen Denkens bei den Griechen, Von Homer bis Hippokrates, Artemis, Zürich and Stuttgart, 1967.

La08. Peter Lax, Mathematics and physics, Bull. Am. Math. Soc. 45/1 (January 2008), 135152.

Li73. Johann Benedict Listing, Über unsere jetzige Kenntniss der Gestalt und Grösse der Erde, Nachr. d. Kgl. Gesellsch. d. Wiss. und der Georg August Univ., Göttingen (5. Februar, No. 3, 1873, pp. 33-98), reprinted September 2007, VDM Verlag, ISBN: 3836422999.

Ma06. Yuri Manin, Presentation to the Closing round table "Are pure and applied mathematics drifting apart?", in: M. Sanz-Solé et al. (eds.), Proceedings of the International Congress of Mathematicians, (Madrid, August 22-30, 2006), European Mathematical Society, Zürich, 2007, pp. 762-764, 775-776.

Ma07. Yuri Manin, Mathematical knowledge: internal, social and cultural aspects. Commissioned for C. Bartocci and P. Odifreddi (eds.), Mathematics and Culture, vol. 2, 2007, math.HO/0703427, also in: Mathematics as Metaphor: Selected Essays of Yuri I. Manin with Foreword by Freeman J. Dyson, American Mathematical Society, 2007, pp. 3-26. Stanley, An Introduction to Econophysics: Correlations and Complexity in Finance, Cambridge University Press, New York, 2000.

Na95. Peter Naur, Knowing and the Mystique of Logic and Rules, Kluwer Academic Publishers, Dordrecht, 1995.

Pe77. Charles Sanders Peirce, The fixation of belief, The Popular Science Monthly (1878), reprinted in: The Collected Papers of Charles Sanders Peirce, Edited by Ch. Hartshorne and P. Weiss, Volume V, book 2, Harvard University Press, Cambridge Mass. 1934, \#358-386, pp. 223-247; http://milne.ruc.dk/\$ \sim\$Booss/MaMoCalc/WP1_Peirce_Fixation.pdf This is the original text by Peirce, but edited for first year students of the Modelling / Calculus Class at Roskilde University.

Sm06. Lee Smolin, The Trouble with Physics, Allen Lane, Penguin Books, London, 2006.

St00. John M. Steele, Observations and Predictions of Eclipse Times by Early Astronomers, Kluwer Academic Publishers, Dordrecht, 2000.

To01. Wolfgang Torge, Geodesy, 3. completely rev. and extended ed., de Gruyter, Berlin-New York, 2001. 